\title{
Fabrication tolerant silicon MZI filter
}

\author{
Sarvagya Dwivedi ${ }^{1}$, Herbert D'heer ${ }^{1}$ and Wim Bogaerts ${ }^{1,2}$ \\ ${ }^{1}$ Photonics Research Group, Department of Information Technology Ghent University—imec, \\ Center for Nano- and Biophotonics, Sint-Pietersnieuwstraat 41, B-9000 Ghent, Belgium \\ ${ }^{2}$ now also with: Luceda Photonics, Dendermonde, Belgium \\ Email: sarvagya.dwivedi@intec.ugent.be
}

\begin{abstract}
A compact fabrication tolerant MZI filter is demonstrated. The measured device shows a 20 -fold improved tolerance to systematic waveguide linewidth variations, with a wavelength shift of less than $60 \mathrm{pm} / \mathrm{nm}$ linewidth change.
\end{abstract}

\section{INTRODUCTION}

One of the most pronounced weaknesses of silicon nanophotonics is the need for extremely precise fabrication, especially for passive wavelength filter devices. The high index contrast and submicron waveguide dimensions will translate any change in waveguide width and height into a strong change in refractive index [1]. When used in an interferometric wavelength filter, a $1 \mathrm{~nm}$ change in width can easily result in a $1 \mathrm{~nm}$ change in the filter response.

While fabrication technology is systematically improving, the required fabrication control will ultimately dictate the yield of larger circuits. Fabrication variations can be actively compensated (e.g. with thermal tuning), but this will drive up power consumption and complicate the device with active control circuitry. Pre-fabrication or post-fabrication trimming [2] of the components can be used to correct the effects of the "last nanometer" fabrication offsets. However it comes with a significantly increased cost of the whole fabrication process.

In this paper we demonstrate a technique to make a wavelength filter tolerant to linewidth variations by design, without changing the fabrication process itself. We show that indeed it is possible to get a fabrication tolerant MZI filter by using different mode confinement in the arms of the filter. The technique can be applied to more complex filters, and extended to include tolerance to other effects, such as waveguide thickness and temperature [3].

\section{DESIGN AND FABRICATION}

The most simple wavelength filter we can construct is the Mach-Zehnder interferometer (MZI). The device, pictured in Fig. 2, uses a shallow-etch multimode interference coupler (MMI) as a 50/50 $1 \times 2$ power splitter, and the same is used as a combiner [4]. The sensitivity of the spectrum position to width deviation $d \lambda / d w$ for a conventional MZI implemented in 450 $\mathrm{nm} \times 220 \mathrm{~nm}$ strip waveguides [1] with air top cladding at a wavelength of $1550 \mathrm{~nm}$ is shown in Fig. 1. The device has

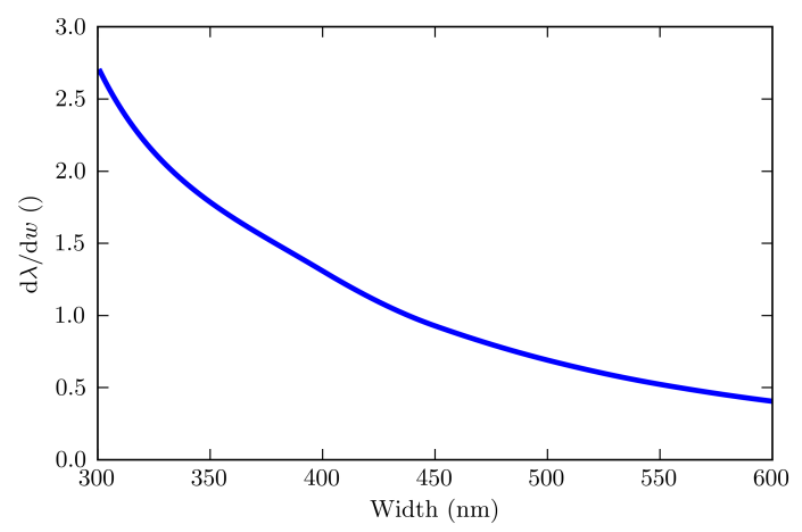

Fig. 1. Sensitivity of spectrum position to width deviation, $\frac{d \lambda}{d w}$, for conventional MZI with air top cladding at $1550 \mathrm{~nm}$.

around $1 \mathrm{~nm}$ wavelength shift for a $1 \mathrm{~nm}$ change in waveguide width. The graph also shows that this sensitivity decreases for larger width since the mode confinement in a wider waveguide is larger and converges to that of the slab waveguide. The shift of the spectrum due to a width deviation of -2 and $+2 \mathrm{~nm}$ for this MZI is shown in Fig. 3(a) for a designed free spectral range (FSR) of $5 \mathrm{~nm}$.

Basically, we can write the wavelength sensitivity of the device to any parameter $X$ as

$$
\frac{d \lambda}{d X}=\frac{\lambda_{m}}{n_{g}} \frac{d n_{e f f}}{d X}
$$

where $X$ could be replaced by the waveguide width $(w)$, height $(h)$, temperature or any other influence. $\lambda_{\mathrm{m}}$ is the center wavelength and, $\mathrm{n}_{\mathrm{g}}$ is the group index.

To compensate for this effect in our device we use different waveguide widths in the 2 arms, introducing not a single set of optical parameters, but two. The modified expression for the sensitivity becomes

$$
\frac{d \lambda}{d X}=\frac{1}{M}\left(L_{1} \frac{d n_{e f f 1}}{d X}-L_{2} \frac{d n_{e f f 2}}{d X}\right) \rightarrow 0
$$

which we now try to minimize. $M$ is the modified interference order which takes into account the dispersion for a given FSR of the filter. For a device tolerant to width variations the condition for zero sensitivity becomes

$$
\frac{L_{1}}{L_{2}}=\frac{\frac{d n_{e f f 2}}{d w}}{\frac{d n_{e f f 1}}{d w}} \mid \lambda_{0}
$$


It means that, for the filter to be width tolerant the length ratio of the arms should be inversely proportional to the change in effective refractive index of the waveguides of the corresponding arms. The assumption we make here is that the linewidth variation induced by the fabrication process is similar for the waveguides in the two arms. This is generally true if the linewidth change is caused by a lithographic dose variation or a change in etch time, and the device is sufficiently compact.

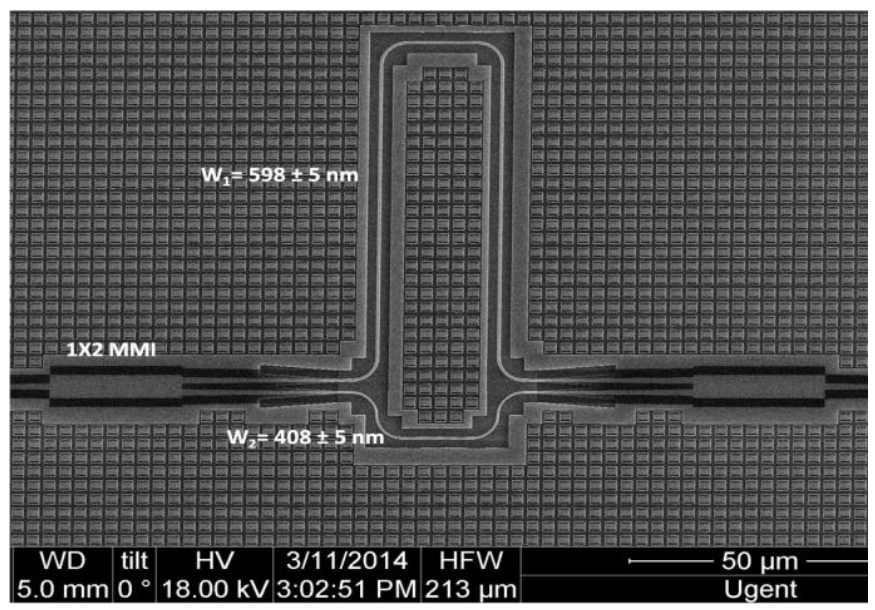

Fig. 2. SEM image of fabricated MZI filter with shallow etch MMI.

\section{EXPERIMENT}

We designed the devices using the IPKISS parametric design framework [5]. We chose a nominal linewidth of $400 \mathrm{~nm}$ in the lower arm and $600 \mathrm{~nm}$ in the upper arm of the MZI. To demonstrate the tolerant behavior we replicated the device with deliberate variations in the linewidth in both arms over a $20 \mathrm{~nm}$ range: $\mathrm{A} \pm 10 \mathrm{~nm}$ width variation can be considered as controllable for an optimized, stabilized fabrication process. The simulation of this device is plotted in Fig.3 (b), and to compare the response a conventional, non-compensated MZI is plotted in Fig.3 (a), but with only a $\pm 2 \mathrm{~nm}$ linewidth change. The simulations were performed using the circuit simulator Caphe [6].

The devices were then fabricated in IMEC's standard passive platform using the ePIXfab MPW service. This process uses $200 \mathrm{~mm}$ SOI wafers with nominally $220 \mathrm{~nm}$ of silicon and 2 $\mu \mathrm{m}$ of buried oxide. The measured thickness of the silicon waveguide in the presented devices is $218 \pm 3 \mathrm{~nm}$. The measured arm width is $598 \pm 5 \mathrm{~nm}$ in the upper arm and $408 \pm 5$ $\mathrm{nm}$ in the lower arm approximately, as shown in SEM image of device in Fig. 2. The calculated length of the upper and lower arm is $172 \mu \mathrm{m}$ and $45 \mu \mathrm{m}$ respectively.

The experimental measurements are shown in Fig. 3(c) and matches very well with the simulation. The devices show an extinction ratio of more than $25 \mathrm{~dB}$ and an insertion loss of less than $0.3 \mathrm{~dB}$ throughout the $\mathrm{C}$ band. The measured FSR of the filter is around $5 \mathrm{~nm}$.


Fig. 3. (a) Simulated spectra of a conventional MZI for $0,-2$ and $+2 \mathrm{~nm}$ width offset. (b) Simulated spectra of the optimized design for $0,-10$ and $+10 \mathrm{~nm}$ width offset (c) Measured transmission and (d) $\mathrm{d} \lambda / \mathrm{d} w$ variation with wavelength.

The extracted sensitivity to linewidth $d \lambda / d w$ is shown in Fig.3(d). The measured width sensitivity is reduced from $1 \mathrm{~nm} / \mathrm{nm}$ to less than $60 \mathrm{pm} / \mathrm{nm}$ over the entire spectrum with standard deviation of $15 \mathrm{pm} / \mathrm{nm}$ which is measured over four devices with width offsets of $\pm 5 \mathrm{~nm}$ and $\pm 10 \mathrm{~nm}$.

\section{CONCLUSION}

We demonstrated a compact fabrication tolerant filter on SOI. The measured results show a reduced linewidth sensitivity of less than $60 \mathrm{pm} / \mathrm{nm}$ over a wide band which is an almost 20 fold improvement compared to conventional devices. This technique can also be extended to reduce sensitivity to a number of other effects and ultimately to designs with an intrinsically improved yield.

\section{REFERENCES}

[1] S. Selvaraja, W. Bogaerts, P. Dumon et al., IEEE J. Sel. Top. Quantum Electron. 16(1), p.316 - 324 (2010)

[2] J. Schrauwen, D. Van Thourhout, R. Baets, Opt. Express 16(6), p.3738$3743(2008)$

[3] S. Dwivedi, H. D'heer, W. Bogaerts, IEEE Photon. Technol. Lett. 25(22), p.2167 - 2170 (2013)

[4] W. Bogaerts, S. Selvaraja, P. Dumon et al., J. Sel. Top. Quantum Electron. 16(1), p.33-44 (2010)

[5] W. Bogaerts, Y. Li, S. Pathak et al., Proc. SPIE 8781, p.878102 (2013)

[6] M. Fiers, T. Van Vaerenbergh, K. Caluwaerts et al. J. Opt. Soc. Am. B, 29(5), p.896-900 (2012) 